\title{
Solution of the kinetic equations governing trap filling. Consequences concerning dose dependence and dose-rate effects
}

\author{
R. Chen, ${ }^{*}$ S. W. S. McKeever, ${ }^{\dagger}$ and S. A. Durrani \\ Department of Physics, University of Birmingham, P.O. Box 363, Birmingham B15 2TT, England
}

(Received 12 December 1980; revised manuscript received 23 June 1981)

\begin{abstract}
The equations governing the traffic of charge carriers during the filling, by ionizing radiation, of traps and luminescence centers in an insulator are numerically solved. The numerical solution is that of a set of four simultaneous differential equations governing the time-dependent functions of concentrations of electrons and holes in the conduction and valence bands and in traps and centers. The results are more general and accurate than those reported previously since no assumptions concerning the proximity to equilibrium have to be made. Moreover, all previous calculations took into account the accumulated concentrations at the end of the irradiation, whereas we have considered an additional period of time after the excitation which allows for the relaxation of carriers in the bands. This simulates the experimental conditions more accurately because during this time any charge carriers which may have accumulated in the conduction and valence bands will relax into the traps and centers and, in doing so, will contribute to the final concentration of trapped charge. In our calculations we have allowed for this by letting the charge in the bands decay for a period of time $T$ following the cessation of the irradiation (which occurs at time $t$ ). Thus, the level of trapped charge $n$ is calculated at time $t+T$ and this is taken to be a better representation of the trapped charge density. Results were obtained for very high and very low dose rates (intensities) of the radiation. Experimental findings of the dose dependence of thermoluminescence (TL) are susceptible to analysis by the approach developed by us. By adding a competing trapping level and changing the set of equations appropriately, we get a set of five simultaneous differential equations. In this way we can test the previous approximative results yielding a superlinear filling of one of the traps. It is found that, under an appropriate choice of parameters, superlinearity emerges, although the results are not identical to those of the previous approximations. In addition, an important result to emerge from the analysis is the possible dependence of TL output on the dose rate for a constant total dose. Recent experimental results of such a dependence on TL in quartz are shown to be in general accord with the numerical results.
\end{abstract}

\section{INTRODUCTION}

The irradiation of semiconductors or insulators with ionizing radiation at suitably low temperatures gives rise to the accumulation of trapped charge carriers at localized energy levels within the material's forbidden energy gap. It is the storage of trapped electrons and holes in this manner that allows the observation of thermoluminescence (TL) which is emitted when the material is heated following the cessation of the irradiation. The light emission is the result of radiative recombination of electron-hole pairs which have become freed from their traps as a result of the temperature increase. Thus, it is generally considered that monitoring the increase in TL emission with imparted dose of ra- diation (TL "growth curves") is a convenient means of studying the buildup of trapped charge in luminescent phosphors.

There have been several attempts to analyze the kinetics of charge storage in phosphors with a view to extracting reliable information concerning the charge-storage parameters from experimentally produced growth curves. $^{1-9}$ Usually, these methods proceed by introducing certain simplifying assumptions and approximations into the differential equations which describe the generation, trapping, and recombination of charge during the irradiation. In this paper, by comparing the approximate analyses with the more exact numerical solutions of the said differential equations, we show that the approximate solutions are somewhat res-

(C) 1981 The American Physical Society 
trictive in that they are valid for only a part of the range of feasible parameters. The numerical solutions presented here reveal a wider variety of growth-curve shapes and magnitudes, and introduce the possibility of the dependence of the trapped charge concentration on radiation intensity-i.e., the rate at which the ionizing radiation was initially delivered.

\section{NUMERICAL ANALYSIS}

In a series of papers, ${ }^{2-7}$ Maxia and co-workers have discussed the filling of traps under a given intensity of an ionizing radiation. These workers present a set of four simultaneous differential equations (see below) governing the traffic of electrons and holes generated by irradiation, in the conduction and valence bands and in traps and centers, respectively. The four equations present the relationships between the number (per unit volume) of electrons in the conduction band $n_{c}$, holes in the valence band $n_{v}$, electrons in traps $n$, and holes in centers $n_{h}$ - all as functions of time. Maxia ${ }^{6,7}$ makes the quite plausible assumption that the excitation of the sample is carried out when the temperature of the specimen is low enough so that trapped electrons and holes are not raised thermally to the conduction and valence bands, respectively, during excitation. Even after making this simplifying assumption, Maxia considers the remaining set of equations to be too difficult to solve, even numerically, and prefers to reach conclusions by using the criteria of a minimum rate of entropy production. ${ }^{4,5}$ Using this principle, Maxia ${ }^{6,7}$ gets

$$
\frac{d n_{c}}{d t}=\frac{d n_{v}}{d t}=\frac{1}{2} \gamma \phi
$$

where $\phi\left(\mathrm{erg} \mathrm{s}^{-1} \mathrm{~cm}^{-2}\right)$ is the incident flux of ionizing radiation energy and $\gamma\left(\mathrm{erg}^{-1} \mathrm{~cm}^{-1}\right)$ is the efficiency factor for pair production. [Note that later we shall use different notations, namely, the dose rate $r\left(\operatorname{rad~s} s^{-1}\right)$ and the efficiency of electron-hole generation $k\left(\mathrm{~cm}^{-3} \mathrm{rad}^{-1}\right)$; the products $\gamma \phi$ and $k r$ are, however, identical, both having units of $\mathrm{cm}^{-3}$ $\mathrm{s}^{-1}$.] In the discussion of his approach, Maxia ${ }^{7}$ emphasizes, however, that the assumptions made are somewhat limiting and that, in fact, after a certain length of time the free-carrier densities reach steady state, namely

$$
\frac{d n_{c}}{d t}=\frac{d n_{v}}{d t}=0 \text {. }
$$

In this range of steady state the contribution to the traps and centers changes from $\gamma \phi / 2$ to $\gamma \phi$. It is not clear when this transition occurs or what behavior takes place in this transition region. Also, in Maxia's derivation of the dose-dependence result, a term quadratic in $\gamma \phi$ is neglected as compared to the term linear in $\gamma \phi$. This may not be accurate for high values of $\phi$ and may limit the generality of Maxia's conclusions.

In the present work we tackle the same problem in a different way. By utilizing a Runge-Kutta sixth-order predictor-corrector program for the solution of simultaneous differential equations, we solve directly, without the use of approximations, the set of four (or five, see below) equations for given sets of the relevant parameters. In the calculation we initialize the concentrations of trapped and free charge carriers to zero and then follow the changes in occupancy of traps, centers, and conduction and valence bands over a series of small time intervals. By keeping an account of the carrier concentrations the buildup of trapped charge may be monitored over the whole of the irradiation period $t$. The calculation was performed on a CDC 7600 computer.

An important point that we have taken account of in the computation is the following. Suppose that an irradiation takes place for a period of time $t$ and that at the end of this period there are electrons left in the conduction band and holes in the valence band. These concentrations of free carriers would relax into the traps and centers in the time following the irradiation and prior to the "readout" process (namely, heating in the case of thermoluminescence). This relaxation will add charge carriers to the traps and centers involved. The effect of this addition would be particularly important when high excitation dose rates (intensities) and short irradiation times are utilized when the accumulation of free charge carriers is expected to be high.

In order to simulate this situation in the computation, we have continued the calculation beyond the end of the "irradiation" time for an extra length of time $T$. For this latter calculation we set the irradiation intensity $f$ to zero at time $t$ and calculate values of $n(t+T)$. We take $n(t+T)=n(t)$ at $T=0$. In our computations we usually took $T=50 \mathrm{~s}$. This is an arbitrary value but one which we feel is sufficiently long to allow for relaxation of the free charge carriers. Normally one would expect free-carrier lifetimes to be very much smaller than this (subject to the values of the actual transition probabilities and trap and center concentrations). In one or two of our calculations, however, 
$50 \mathrm{~s}$ was not enough to allow for sufficient relaxation. In all cases, at the end of the extra time $T=50 \mathrm{~s}$, we check the values of $n_{c}(t+T)$ and $n_{v}(t+T)$ and accept the results only if they are negligible as compared to the final occupancies $n(t+T)$ and $n_{h}(t+T)$, respectively. We believe that the concentrations $n(t+T)$ and $n_{h}(t+T)$ are more accurate monitors of the actual TL emission than their counterparts $n(t)$ and $n_{h}(t)$.

\section{KINETIC EQUATIONS}

The possible existence of several traps and/or recombination centers for both electrons and holes means that, in practice, there is a wide variety of situations that could be considered in order to describe the energy-level scheme of a particular phosphor. To begin with, let us consider one of the simplest energy-level schemes, namely, that of one trap and one recombination center ("one trapone center") shown in Fig. 1. The notation is somewhat different from that of Maxia ${ }^{6,7}$. The magnitudes involved are: $n_{c}$-concentration of electrons in the conduction band $\left(\mathrm{cm}^{-3}\right) ; n_{v}$ concentration of holes in the valence band $\left(\mathrm{cm}^{-3}\right)$; $n$-concentration of electrons in traps $\left(\mathrm{cm}^{-3}\right)$; $N$-concentration of the traps $\left(\mathrm{cm}^{-3}\right) ; n_{h}-$ concentration of holes in centers $\left(\mathrm{cm}^{-3}\right) ; N_{h}-$ concentration of hole centers $\left(\mathrm{cm}^{-3}\right) ; A$-transition probability of electrons from the conduction band to the traps $\left(\mathrm{cm}^{3} \mathrm{~s}^{-1}\right) ; A_{h}$-transition probability of holes from the valence band into the centers $\left(\mathrm{cm}^{3} \mathrm{~s}^{-1}\right) ; A_{R}$-recombination probability of electrons from the conduction band with holes in centers $\left(\mathrm{cm}^{3} \mathrm{~s}^{-1}\right) ; f$-the electron-hole generation

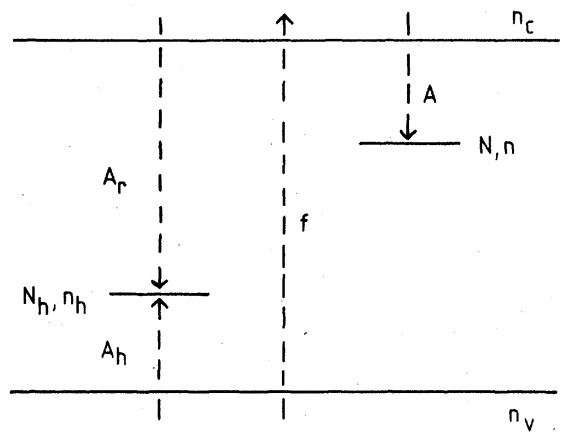

FIG. 1. The energy-level scheme used in this paper for the simple case of one trap and one recombination center ("one-trap - one-center"). The arrows indicate the allowed transitions and the parameters indicated in the diagram are defined in the text. rate $\left(\mathrm{cm}^{-3} \mathrm{~s}^{-1}\right)$; and $R$-the total electron-hole generation $\left(\mathrm{cm}^{-3}\right)$, proportional to the total dose.

In this energy-level scheme we allow for the generation of free electrons and holes, trapping of free electrons, and recombination of free electrons with previously trapped holes. We assume, in the same way as Maxia, ${ }^{6,7}$ that charge carriers are not raised thermally to the respective bands during excitation. This can always be ensured experimentally by performing the irradiation at a temperature low enough as compared to that of the TL peaks in question, and most of the experiments of thermally stimulated phenomena are indeed performed in this way. We also assume that no band-to-band or trap-to-trap recombinations are possible, or rather - which is generally accepted - that the probabilities for such recombinations are very low. We also assume that the recombination of holes from the valence band with trapped electrons is negligible. This is not necessarily always the case. However, in order to demonstrate, say, the occurrence of a dose-rate effect (see below), the recombination of free electrons with trapped holes at the center suffices. It is to be emphasized that the numerical solution of the more general equations including thermal stimulation during the irradiation, and recombination at the trap [see Eqs. (1) - (4) in Ref. 2] could be carried out with no particular additional difficulty. However, the number of parameters involved would then increase substantially, with a corresponding increase in the computation time, and we have preferred to demonstrate the variety of possible relationships which can be obtained in the simplest possible framework. A note should be made concerning the total generation $R$, the generation rate $f$, and their relation to the total dose and the dose rate. If $f$ is constant during the irradiation, obviously $R=f t$. If $f$ is not constant, one should take $R=\int_{0}^{t} f d t$, but if this is the case, the final result as a function of $R$ may not be unambiguously defined since different relationships (between trapped charge and dose rate) may occur during different parts of the irradiation time. As briefly mentioned above, the dose rate $r\left(\mathrm{rad} \mathrm{s}^{-1}\right)$ is related to the generation rate $f$ by $f=k r$ where $k$ is the ionization efficien$c y$, a constant (for a given material) with units of $\mathrm{cm}^{-3} \mathrm{rad}^{-1}$. The same constant obviously relates the total dose imparted $D$ with the total generation $R$, i.e., $R=k D$.

If one assumes that the electrons raised to the conduction band will necessarily be accumulated in the trap and the holes in the center, one certainly 
expects at the end of the irradiation to have $R$ electrons in the trapping state and $R$ holes in the recombination center. Since these are dose rate (intensity) independent, the resulting TL should be expected to be dose rate independent as well. It may be that behind the concept that dose rate effects are, in a way, paradoxical, lies the belief in such a simple model for TL excitation. However, in the framework of "one-trap - one-center" we now add another element, namely, that electrons raised into the conduction band can recombine, during the irradiation, with holes already trapped in the recombination center (transition denoted by $A_{r}$ in Fig. 1). This assumption is very plausible since this is the same type of transition which later, during the heating, produces the TL emission. We are interested in the smaller of the final concentrations of electrons in traps $(n)$, and holes in centers $\left(n_{h}\right)$ following irradiation. ${ }^{10}$ In this simple model, since electrons and holes are produced in pairs and annihilate in pairs, the final values of $n$ and $n_{h}$ will be equal; thus, either of them represents the minimum. The final values of $n$ and $n_{h}$ would, however, not have been necessarily equal had we allowed for the existence of another electron or hole reservoir.

The four simultaneous differential equations governing the excitation process in the "one-trap - one-center" case are (see Fig. 1)

$$
\begin{aligned}
& \frac{d n_{c}}{d t}=f-n_{c} A_{r} n_{h}-n_{c}(N-n) A, \\
& \frac{d n}{d t}=n_{c}(N-n) A, \\
& \frac{d n_{v}}{d t}=f-n_{v}\left(N_{h}-n_{h}\right) A_{h}, \\
& \frac{d n_{h}}{d t}=n_{v}\left(N_{h}-n_{h}\right) A_{h}-n_{c} n_{h} A_{r} .
\end{aligned}
$$

In this model, the system involves the functions $n(t), n_{c}(t), n_{h}(t)$, and $n_{v}(t)$ and the constant trapping parameters $N, N_{h}, A, A_{r}$, and $A_{h}$. Additional parameters are the electron-hole generation rate $f$ and the length of time of irradiation $t$ (or alternatively, the total generation $R$ ). For a given set of the sample paramaters, the values of $f$ and $t$ or $R$ can be changed in order to see the behavior as a function of the dose rate or of the total dose.

\section{RESULTS}

\section{A. One-trap - one-center}

A growth curve of trapped charge population against total generation $R$ is seen in Fig. 2. The values of the parameters used in this calculation

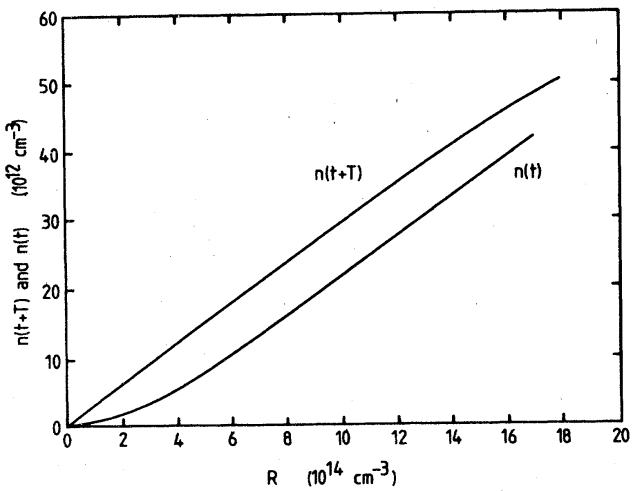

FIG. 2. Growth of $n(t+T)$ and of $n(t)$ with total generation $R$ for the one-trap - one-center case. The importance of the additional time $T$ at the end of the irradiation for relaxation of free charge carriers is indicated by the fact that $n(t+T)>n(t)$. In addition $n(t)$ is seen to exhibit superlinear growth while $n(t+T)$ does not. The parameters chosen in this figure are the following: $N=10^{15} \mathrm{~cm}^{-3} ; N_{h}=3 \times 10^{14} \mathrm{~cm}^{-3} ; A=10^{-17}$ $\mathrm{cm}^{3} \mathrm{~s}^{-1} ; A_{r}=10^{-13} \mathrm{~cm}^{3} \mathrm{~s}^{-1} ; A_{h}=10^{-15} \mathrm{~cm}^{3} \mathrm{~s}^{-1}$; $f=10^{14} \mathrm{~cm}^{-3} \mathrm{~s}^{-1}$.

are listed in the figure caption. The parameter values chosen were such that, in this particular case, there is a considerable difference between the values of $n(t+T)$ and $n(t)$, thus illustrating the importance of the extra time $T$ in the determination of the final population of trapped charge. The fact that $n(t+T)>n(t)$ means, in effect, that free charge has accumulated in the conduction band during the period of irradiation. (Note, however, that we do not make any statement on the closeness of this charge population to equilibrium.) This in turn means that those analyses ${ }^{2,3,8,9}$ which assume that $d n_{c} / d t<<d n / d t$ throughout the irradation period are not appropriate in this case (for, in these analyses, the accumulation of free charge in the bands should be negligible). Nonequilibrium thermodynamics ${ }^{4-7}$ might, therefore, appear to present a better alternative, but it must be remembered that in the numerical analysis we do not determine whether the accumulated population of free charge is in equilibrium or not. It suffices to say that it may be non-negligible. Under these circumstances the decay of this free charge population into traps and centers at the end of the irradiation must be taken into account. In this respect, even those treatments which allow for accumulation of free charge in the bands, albeit under nonequilibrium kinetics, ${ }^{4-7}$ are also inadequate because they do not allow for the relaxation of this charge following the end of the irradiation.

The difference between $n(t+T)$ and $n(t)$ is not 
just quantitative. Figure 2 clearly shows a difference in the shape of the growth curves, such that $n(t)$ exhibits superlinear growth but $n(t+T)$ does not. In fact, in all of the cases that we have tried where $n(t+T)>n(t)$ in the one-trap - one-center scheme, $n(t)$ always exhibits superlinear growth but we have never observed such behavior in $n(t+T)$.

It ought to be remembered that some sets of values of the relevant parameters produced the result $n(t+T) \simeq n(t)$. This was particularly so for low values of $f$. Under these circumstances (i.e., low $f$ ) the growth curve [identical for both $n(t)$ and $n(t+T)$ ] will not exhibit superlinearity, and the condition $d n_{c} / d t<<d n / d t$ could be said to have been met.

To obtain Fig. 2, the generation rate $f$ was held constant and the total irradiation time $t$ varied in order to produce the dependence of the trapped charge concentration upon total generation $R$. Equations (3) - (6) can also be solved by holding $R$ constant and varying $f$. In this way any dependence of the trapped charge population upon $f$ can be looked for. A typical set of results is given in Fig. 3. Here we see a family of curves in which the variation of $n(t+T)$ with generation rate $f$ is monitored for different total generations $R$. As can be seen from the figure, $n(t+T)$ is nonconstant with $f$ and indeed each curve exhibits a region in which the trapped charge concentration either decreases or increases as $f$ increases. The nature of the dependency (i.e., increase or decrease) depends upon the value of $R$, and the range of $f$ over which $n(t+T)$ is nonconstant increases as $R$ increases.

A consequence of the dependence of $n(t+T)$ upon generation rate is that the growth curve will also be dependent upon $f$. This can be seen in Fig. 4 where we have plotted the growth of $n(t+T)$ with $R$ for three different values of $f$. For the particular sets of parameters chosen here the efficiency of trap filling, for a given total dose, can be seen to decrease as the generation rate increases.

The nature and strength of the dose rate dependency was found to be sensitive to the values of certain of the characteristic parameters. This was found to be particularly so for $N_{h}$, the concentration of available hole centers. Figure 5 shows the variation of $n(t+T)$ with $f$ for different values of $N_{h}$. For some ranges of $N_{h}$ (e.g., $10^{18}-10^{17}$ in the example shown) the value of this parameter has little effect on the nature of the dependency. However, in a different range (e.g., $3 \times 10^{15}-2 \times 10^{15}$ ) the dependency is found to be highly sensitive to $N_{h}$. Indeed, the dependency can be seen to change from one of decreasing $n(t+T)$ with increasing $f$, to one of increasing $n(t+T)$ with increasing $f$.

The essential ingredient which appears to be necessary in order to generate a dependence of trapped charge population upon radiation intensity is the occurrence of recombination during the irradiation period. As we have already stated, dose rate-dependent effects will not be observed if recombination during irradiation is not allowed. Thus the high sensitivity shown by the variation of $n(t+T)$ with $f$ to the value of $N_{h}$ may be a re-

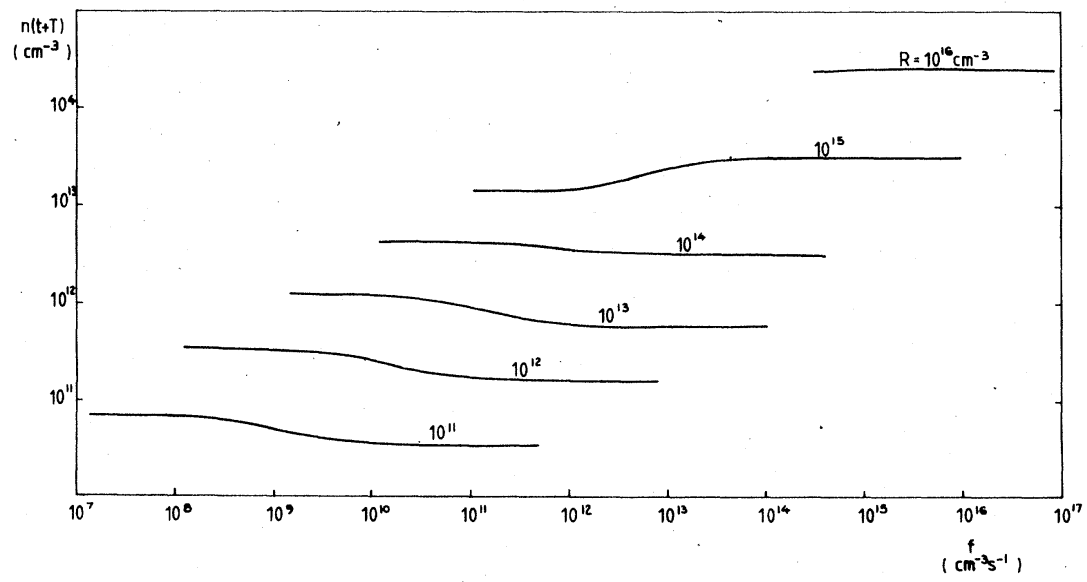

FIG. 3. The dependence of $n(t+T)$ upon $f$ for different values of $R$. Each curve exhibits a region in which $n(t+T)$ is nonconstant with $f$. The range of $f$ over which $n(t+T)$ is nonconstant increases as $R$ increases. In some cases, $n(t+T)$ is seen to decrease with increasing $f$, but as $R$ is increased this behavior changes to one of increasing $n(t+T)$ with increasing $f$. The values of the parameters used in this figure are the following: $N_{h}=3 \times 10^{14} \mathrm{~cm}^{-3}$; $N=10^{15} \mathrm{~cm}^{-3} ; A=10^{-17} \mathrm{~cm}^{3} \mathrm{~s}^{-1} ; A_{r}=10^{-13} \mathrm{~cm}^{3} \mathrm{~s}^{-1} ;$ and $A_{h}=10^{-15} \mathrm{~cm}^{3} \mathrm{~s}^{-1}$. 


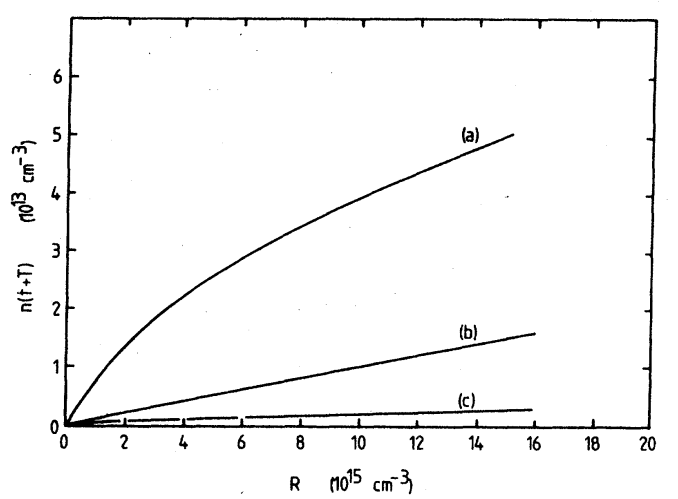

FIG. 4. Growth of $n(t+T)$ for three values of $f$, namely (a) $10^{13} \mathrm{~cm}^{-3} \mathrm{~s}^{-1}$; (b) $10^{15} \mathrm{~cm}^{-3} \mathrm{~s}^{-1}$; and (c) $10^{17} \mathrm{~cm}^{-3} \mathrm{~s}^{-1}$. For the set of parameters chosen, the efficiency of trap filling decreases as the generation rate increases. The chosen parameters are the following: $N=10^{15} \mathrm{~cm}^{-3} ; N_{h}=10^{17} \mathrm{~cm}^{-3} ; A=10^{-17} \mathrm{~cm}^{3} \mathrm{~s}^{-1}$; $A_{r}=10^{-13} \mathrm{~cm}^{3} \mathrm{~s}^{-1}$; and $A_{h}=10^{-15} \mathrm{~cm}^{3} \mathrm{~s}^{-1}$.

\section{flection of this fact.}

It should be noted that for the parameters in Fig. 5, the corresponding values of $n(t)$ were always observed to decrease with increasing $f$. At large values of $N_{h}$, when recombination can be expected to be high (subject to the values of $A_{h}$ and $\left.A_{r}\right)$ we find that the value of $n(t+T)$ tends to follow the behavior of $n(t)$ more closely than when $N_{h}$ has a relatively low value. This would appear to be because more free electrons relax via the

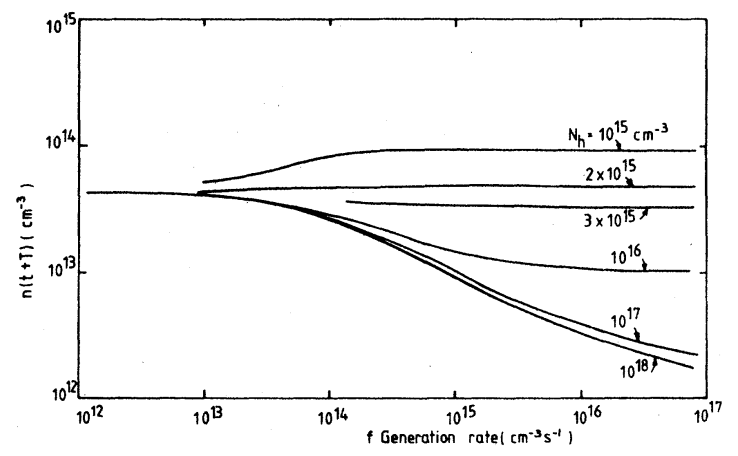

FIG. 5. Variation in $n(t+T)$ with generation rate $f$ for different values of $N_{h}$. At very low and very high values of $f, n(t+T)$ is essentially independent of the generation rate. However, there exists a range of $f$ over which $n(t+T)$ can decrease, increase, or remain independent of $f$, depending upon the values of $N_{h}$. The parameters used in this diagram are the following: $N=10^{15} \mathrm{~cm}^{-3} ; A=10^{-17} \mathrm{~cm}^{3} \mathrm{~s}^{-1} ; A_{\mathrm{r}}=10^{-13} \mathrm{~cm}^{3} \mathrm{~s}^{-1}$; $A_{h}=10^{-15} \mathrm{~cm}^{3} \mathrm{~s}^{-1}$; and $R=10^{16} \mathrm{~cm}^{-3}$. recombination center than are trapped in the electron traps during the period following irradiation. This, in effect, means that, despite the fact that charge does accumulate in the bands, $n(t+T)$ $\simeq n(t)$. This serves to illustrate that equality between $n(t+T)$ and $n(t)$ should not necessarily be taken as an indication that $d n_{c} / d t<<d n / d t$ during the irradiation period. [This in turn opens up the possibility of obtaining a superlinear growth curve for $n(t+T)$ for the one-trap - one-center case. It must be said, however, that for the sets of parameters that we have tried, we have not observed such a situation; we have obviously not exhausted all of the possible variations.]

At smaller values of $N_{h}$ we can expect that a much larger proportion of the accumulated freeelectron population will decay via the electron traps, thus adding a contribution which opposes the dose rate dependency of $n(t)$. In fact, for a value of $N_{h}$ between $2 \times 10^{15}$ and $3 \times 10^{15}$, the two effects appear to cancel producing a dose rate independent $n(t+T)$. At even lower values of $N_{h}$, the final result yields an increase in $n(t+T)$ with $f$. This discussion illustrates the importance of recombination during irradiation.

Maxia $^{4-7}$ has considered the kinetics of charge storage in insulators by introducing simplifying assumptions into the differential equations (1)-(4). Most importantly, the concept of nonequilibrium thermodynamics is introduced into the process of charge storage. The important point made here was that $d n_{c} / d t=\frac{1}{2} f$ during the excitation period. Using this assumption, Maxia arrives at

$$
\left(1-\frac{A_{r}}{A}\right) n-\frac{A_{r}}{A} N \ln \left(1-\frac{n}{N}\right)=\frac{1}{2} R,
$$

which describes the buildup of trapped electrons as a function of total generation. Alternatively, by assuming quasiequilibrium throughout the excitation period - i.e., $d n_{c} / d t<<d n / d t$ - Aramu et al. arrived at the following equation:

$$
\left[1-\frac{A_{r}}{A}\right] n-\frac{A_{r}}{A} N \ln \left[1-\frac{n}{N}\right]=R,
$$

which differs from Eq. (7) by the factor of $\frac{1}{2}$ on the right-hand side.

Equations (7) and (8) describe the growth of $n(t)$ with $R$ and it is necessary for us to consider how these approximate solutions compare with the nonapproximative numerical solutions. To do this 
it is important to realize that in assuming $d n_{c} / d t=\frac{1}{2} f$, Eq. (7) allows for the accumulation of electrons in the conduction band. Thus, at the end of the irradiation there may be a considerable concentration of charge $\left[n_{c}(t)\right]$ in the conduction band, and this may be comparable to, or larger than, $n(t)$. Here we restate a point already made, namely, that the approximate solution does not allow for relaxation of $n_{c}(t)$ after the irradiation ceases, thus Eq. (7) considers $n(t)$ only, not $n(t+T)$. Equation (8) also considers $n(t)$ only, but in this case $d n_{c} / d t<<d n / d t$, thus the accumulation of electrons in the conduction band $n_{c}(t)$ may be very small and thus we might expect that $n(t)$ in the approximate solution may be comparable to $n(t+T)$ in the numerical solution. However, this consideration fails to take into account the dependence of $n(t+T)$ upon $f$. Both Eqs. (7) and (8) do not account for any dependence of the trapped charge concentration upon the generation rate.

The situation is best illustrated by plotting the trapped charge concentration against generation rate, as in Fig. 6. Here we see that at low $f$ the numerical solution shows that $n(t)=n(t+T)$. This is because at low generation rates there has

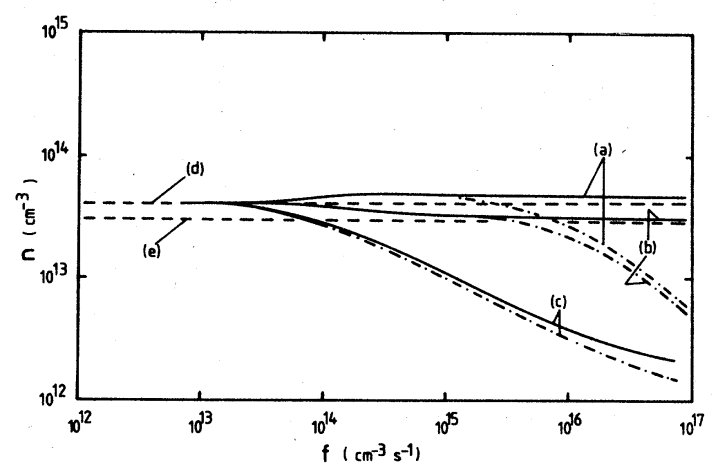

FIG. 6. The variation of $n(t+T)$ (full lines) and $n(t)$ (dot-dash lines) with $f$ for the parameters listed in Fig. 4 , with $R=10^{16} \mathrm{~cm}^{-3}$. The curves corresponding to three different values of $N_{h}$ are shown, namely (a) $2 \times 10^{15} \mathrm{~cm}^{-3}$; (b) $3 \times 10^{15} \mathrm{~cm}^{-3}$; (c) $10^{17} \mathrm{~cm}^{-3}$. At low values of $f$ the approximate solution of Eq. (8) [dashed line (d)] agrees with the numerical solution. For curve (a), $n(t+T)$ remains approximately independent of $f$ and equal to the solution of Eq. (8). In curve (b), $n(t+T)$ deviates from the solution of Eq. (8) and becomes approximately equal to the solution of Eq. (7) [dashed line (e)]. For curve (c), $n(t+T)$ deviates from the solution of both Eqs. (7) and (8) at higher values of $f$. In all three cases (a), (b), and (c), $n(t)<n(t+T)$ at high $f$. been very little accumulation of free charge in the conduction band during irradiation. Under these circumstances we expect good agreement between the solution of Eq. (8) [curve (d)] and the numerical solution, and this is what is observed. Equation (7) [curve (e)] on the other hand gives a value of $n$ which is too low in this region.

However, as $f$ increases, $n(t+T)$ becomes larger than $n(t)$, indicative of the fact that charge is now allowed to accumulate in the conduction band at these values of $f$. Thus, with $N_{h}=10^{17}$ [curve (c)], for example, both $n(t+T)$ and $n(t)$ deviate from the solution predicted by Eq. (7).

However, for $N_{h}=2 \times 10^{15} \mathrm{~cm}^{-3}$ [curve (a)], $n(t+T)$ is seen to remain approximately independent of $f$, such that it remains almost equal to the approximate solution for all $f$. Note though, that this does not mean that the approximations in Eq. (8) are valid throughout this range of generation rates. This is because $n(t+T)>n(t)$, which means that charge definitely is accumulating in the conduction band. Similarly, if we choose $N_{h}=3 \times 10^{15} \mathrm{~cm}^{-3}$ [curve (b)], the solution of Eq. (7) now approximates to the value of $n(t+T)$ at the high generation rates. However, this again does not mean that the approximation involved in Eq. (7) (namely, $d n_{c} / d t=\frac{1}{2} f$ ) is valid in this range of values.

In both these last two cases, values of the parameters were found for which $n(t+T)$ was coincidentally approximately equal to the solution either of Eq. (7) or of Eq. (8). However, in only one situation (namely at low $f$ ) can we say that one of the approximations [i.e., Eq. (7)] compared favorably with the numerical analysis.

\section{B. One-trap - two-centers}

The results of the numerical solution of the differential equations for the case of one trap and one center have illustrated some important points which are worthy of a summary at this juncture. Firstly, it has been shown that it is essential to take into account the relaxation of free charge carriers following the end of the irradiation period. Secondly, in some circumstances the storage of trapped charge during the irradiation is seen to be dependent upon the dose rate; the important factor being the degree of recombination taking place during the excitation. Thirdly, those methods which seek to solve the differential equations by the introduction of assumptions, are found to be applicable only in special instances. With these lessons in 
mind we now extend the analysis to a more complex energy-level scheme.

We have seen (cf. Fig. 5) that $n(t+T)$ can either increase or decrease with increasing $f$, depending upon the values of the relevant parameters. This dose rate dependency broadly agrees with the experimental findings made recently by Groom et al. ${ }^{11}$ who observed dose rate-dependent effects in the TL of natural quartz crystals (see Sec. V). However, more detailed work on the TL of quartz by Valladas and Ferreira ${ }^{12}$ has shown that while one component of the TL emission decreases with increasing dose rate, another increases with increasing dose rate. This result cannot immediately be explained by the results presented so far in this paper which can account for one type of behavior, or the other, but not both in the same sample at the same time. Clearly, the energy-level scheme so far discussed is too simplistic to account for such behavior.

The observations by Valladas and Ferreira were made on different spectral components of the TL emission from quartz and because of this we have introduced an extra recombination center into the simple energy-level scheme. The new energy-level diagram now under consideration is shown in Fig. 7 and the five differential equations which govern the traffic of charge carriers between the trap, centers, and conduction and valence band are

$$
\begin{aligned}
& \frac{d n_{c}}{d t}=f-n_{c} A_{r} n_{h}-n_{c}(N-n) A-n_{c} A_{l l} n_{l}, \\
& \frac{d n}{d t}=n_{c}(N-n) A, \\
& \frac{d n_{v}}{d t}=f-n_{v}\left(N_{h}-n_{h}\right) A_{h}-n_{v}\left(N_{l}-n_{l}\right) A_{l},
\end{aligned}
$$

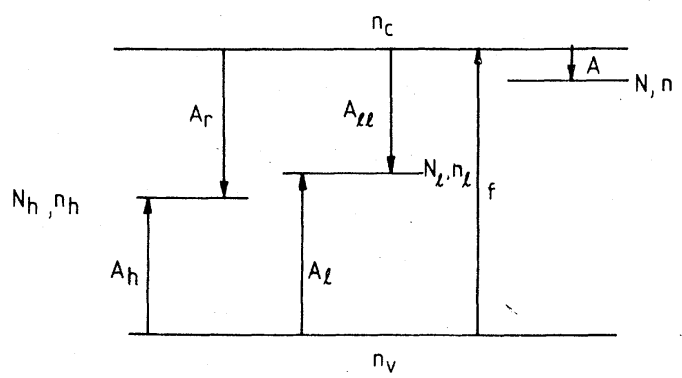

FIG. 7. Energy-level diagram for the one-trap two-centers case. An extra recombination center has been added to the simple energy-level picture of Fig. 1 . The parameters are defined in the text. $\frac{d n_{h}}{d t}=n_{v}\left(N_{h}-n_{h}\right) A_{h}-n_{c} A_{r} n_{h}$,

$\frac{d n_{l}}{d t}=n_{v}\left(N_{l}-n_{l}\right) A_{l}-n_{c} A_{l l} n_{l}$,

where the definitions of the parameters are the same as before with the addition of: $n_{l}$ - concentration of holes in the additional centers $\left(\mathrm{cm}^{-3}\right)$; $N_{l}$-concentration of additional centers $\left(\mathrm{cm}^{-3}\right)$; $A_{l}$-transition probability of holes from the valence band into the additional centers $\left(\mathrm{cm}^{3} \mathrm{~s}^{-1}\right)$; and $A_{l l}$-recombination probability of electrons from the conduction band with holes in the additional centers $\left(\mathrm{cm}^{3} \mathrm{~s}^{-1}\right)$.

We can now attempt to solve Eqs. (8) - (13) numerically with a view to obtaining different behavior for the two recombination centers. (It is to be remembered that TL emission will be proportional to the smaller of the concentrations of electrons in traps and holes in centers: because we have two centers in this case, the limiting concentrations will be that of the holes in the centers.)

Figure 8 shows the results of $n_{h}(t+T)$ and $n_{l}(t+T)$ for the set of parameters given in the figure caption. While $n_{l}$ is seen to increase only by $\sim 5 \%$ as $f$ is increased by a factor of $10^{3}$, the value of $n_{h}$ is seen to increase by a factor of $\sim 6$ over the same range of $f$. This at least illustrates that we

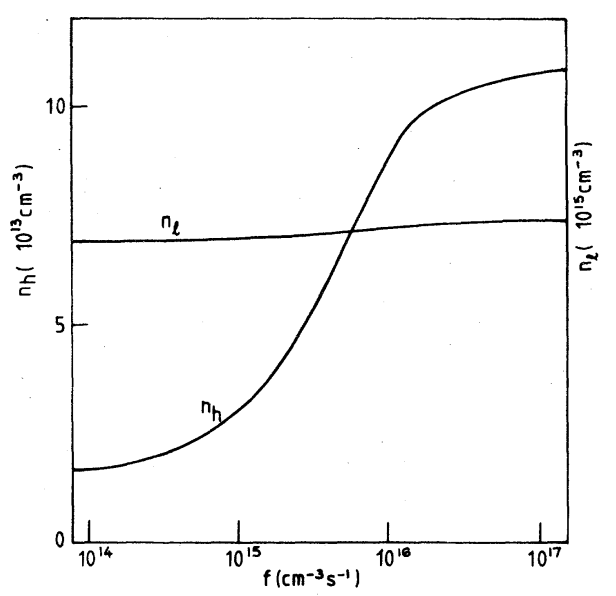

FIG. 8. The final (i.e., at time $t+T$ ) concentrations of holes in the centers as a function of generation rate. Both $n_{h}$ and $n_{l}$ change in the same direction, but $n_{l}$ only increases by a factor of $\sim 5 \%$ compared with an increase by a factor of $\sim 6$ for $n_{l}$. The parameters used are the following: $N=10^{17} \mathrm{~cm}^{-3} ; N_{l}=10^{16} \mathrm{~cm}^{-3}$; $N_{h}=3 \times 10^{14} \mathrm{~cm}^{-3} ; R=10^{16} \mathrm{~cm}^{-3} ; A=10^{-16} \mathrm{~cm}^{3} \mathrm{~s}^{-1}$; $A_{l l}=10^{-15} \mathrm{~cm}^{3} \mathrm{~s}^{-1} ; A_{r}=10^{-15} \mathrm{~cm}^{3} \mathrm{~s}^{-1} ; A_{l}=10^{-15}$ $\mathrm{cm}^{3} \mathrm{~s}^{-1}$; and $A_{h}=10^{-15} \mathrm{~cm}^{3} \mathrm{~s}^{-1}$. 
can expect, in principle, radically different behaviors from different TL components. However, although the strength of the variation is different for the two centers, the nature of the dependency upon $f$ is the same - i.e., both increase with increasing $f$.

Figure 9 shows the results for a different set of parameter values and in this case opposite behavior is seen. In fact, $n_{l}$ decreases by $\sim 17 \%$ whereas $n_{h}$ increases by a factor of $\sim 2$ as $f$ is increased by a factor of $10^{3}$. While we certainly do not claim that these results are optimal in any sense (it is impossible for us to exhaust all of the available combinations of parameter values), it does at least simulate the experimental findings of Valladas and Ferreira. $^{12}$

\section{Two-traps - one-center}

In an attempt to explain the superlinear growth of TL with imparted dose in some luminescent phosphors, Suntharalingam and Cameron ${ }^{13}$ intro-

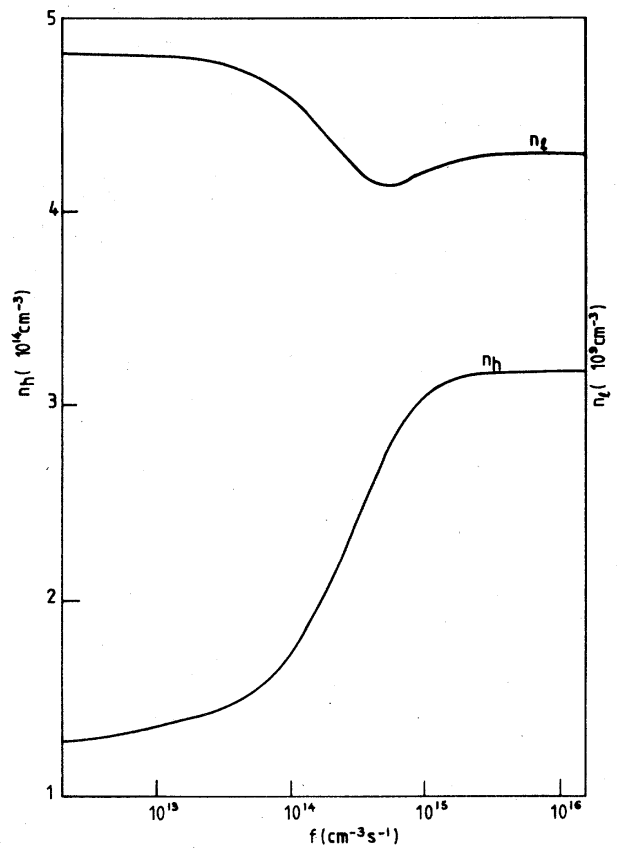

FIG. 9. The final (i.e., at time $t+T$ ) concentrations of holes in the two centers as a function of generation rate. Here $n_{l}$ and $n_{h}$ are seen to change in opposite directions with $n_{h}$ showing an increase by a factor of $>2$ and $n_{l}$ at first decreasing by $\sim 17 \%$, and then slightly increasing. The parameters used are the following: $N_{h}=10^{15} \mathrm{~cm}^{-3} ; N=10^{16} \mathrm{~cm}^{-3} ; A=10^{-16} \mathrm{~cm}^{3} \mathrm{~s}^{-1}$; $A_{r}=10^{-13} \mathrm{~cm}^{3} \mathrm{~s}^{-1} ; R=10^{15} \mathrm{~cm}^{-3} ; N_{l}=10^{13} \mathrm{~cm}^{-3}$; $A_{l l}=10^{-15} \mathrm{~cm}^{3} \mathrm{~s}^{-1} ; A_{l}=10^{-18} \mathrm{~cm}^{3} \mathrm{~s}^{-1}$; and $A_{h}=1.5 \times 10^{-15} \mathrm{~cm}^{3} \mathrm{~s}^{-1}$. duced an extra trap into the simple energy-level picture of one-trap - one-center (cf. Fig. 1). The energy-level scheme considered by these authors is shown in Fig. 10. The "competing trap model" has been investigated by several authors in some detail. The set of differential equations which describes the flow of charge carriers between the traps, center, and conduction and valence bands depicted in Fig. 10, is given below:

$\frac{d n_{c}}{d t}=f-n_{c} A_{r} n_{h}-n_{c}(N-n) A-n_{c}\left(N_{k}-n_{k}\right) A_{k}$,

$\frac{d n}{d t}=n_{c}(N-n) A$

$\frac{d n_{k}}{d t}=n_{c}\left(N_{k}-n_{k}\right) A_{k}$,

$\frac{d n_{v}}{d t}=f-n_{v}\left(N_{h}-n_{h}\right) A_{h}$,

$\frac{d n_{h}}{d t}=n_{v}\left(N_{h}-n_{h}\right) A_{h}-n_{c} n_{h} A_{r}$.

In these equations we have again ignored bandto-band and trap-to-trap recombination, and the thermal release of trapped charge during excitation. The definitions of the terms used in Eqs. (14) - (18) remain the same as those used in Eqs. (2) - (6) with the addition of $n_{k}$ - concentration of trapped electrons in the competing traps $\left(\mathrm{cm}^{-3}\right) ; N_{k}-$ concentration of competing traps $\left(\mathrm{cm}^{-3}\right)$; and $A_{k}$-transition probability of electrons from the conduction band into the competing traps $\left(\mathrm{cm}^{3} \mathrm{~s}^{-1}\right)$.

Recently Chen and Bowman ${ }^{8,9}$ have attempted

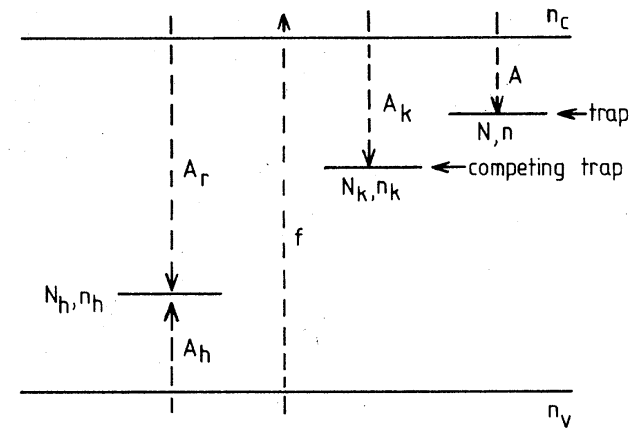

FIG. 10. Energy-level diagram for the two-traps one-center. An extra "competing" trap has been introduced into the simple energy-level scheme of Fig. 1. The definitions of the parameters are listed in the text. 
to solve Eqs. (14) - (18) analytically by introducing some simplifying approximations into the analysis. These authors preferred to use the concept of quasiequilibrium - namely, $d n_{c} / d t$ $<<d n / d t, d n_{k} / d t$ - and thus their analysis is equivalent to that of Aramu et al. ${ }^{2}$ for the onetrap - one-center case. The equation derived by Chen and Bowman to describe the buildup of trapped charge during irradiation is

$$
\begin{aligned}
& N_{k}\left[\frac{A_{r}-A_{k}}{A_{k}}\right]\left[\left(1-\frac{n}{N}\right)^{A_{k} / A}-1\right]+\left(\frac{A-A_{r}}{A}\right) n \\
& -N \frac{A_{r}}{A}\left(\frac{N_{k}}{N}+1\right) \ln \left(1-\frac{n}{N}\right)=R,
\end{aligned}
$$

which is equivalent to Eq. (8) for the one-trap one-center case and can be made the same as Eq. (8) by putting $N_{k}=0$.

The major points to consider about Eq. (19) are that it does not take account of the dependence of the trapped charge concentration upon $f$, nor does it include the additional charge accumulated during relaxation of the free carrier density following the end of the excitation. Equation (19) was derived using the concept of quasiequilibrium throughout the irradiation period, which in turn means negligible free charge accumulation in the conduction band. In a real situation this may or may not be true.

The inadequacy of Eq. (19) to describe the trap filling during excitation for all values of $f$ is best illustrated in Fig. 11. At low values of $f$ there is little charge accumulation in the conduction band and thus the approximate solution gives the same result as the numerical solution, and, of course, $n(t)=n(t+T)$. However, as $f$ increases both $n(t)$ and $n(t+T)$ may deviate from the solution of Eq. (19) (which is independent of $f$ ). In Fig. 11(a) the parameters chosen are such that $n(t+T)$ deviates from the approximate solution as $f$ increases.

However, in Fig. 11(b) the chosen parameters produce the result that $n(t+T)$ is essentially independent of $f$, but is still greater than $n(t)$. A growth curve corresponding to the parameters given in Fig. 11(b) is shown in Fig. 12. The figure indicates very good agreement between the approximation and the numerical result, but this must not be taken as justification of the assumption of quasiequilibrium, because $n(t+T)>n(t)$.

Note that the growth curve for $n(t+T)$ and for Eq. (19) shown in Fig. 12 does not exhibit any superlinearity. Using Eq. (19), Chen and Bowman

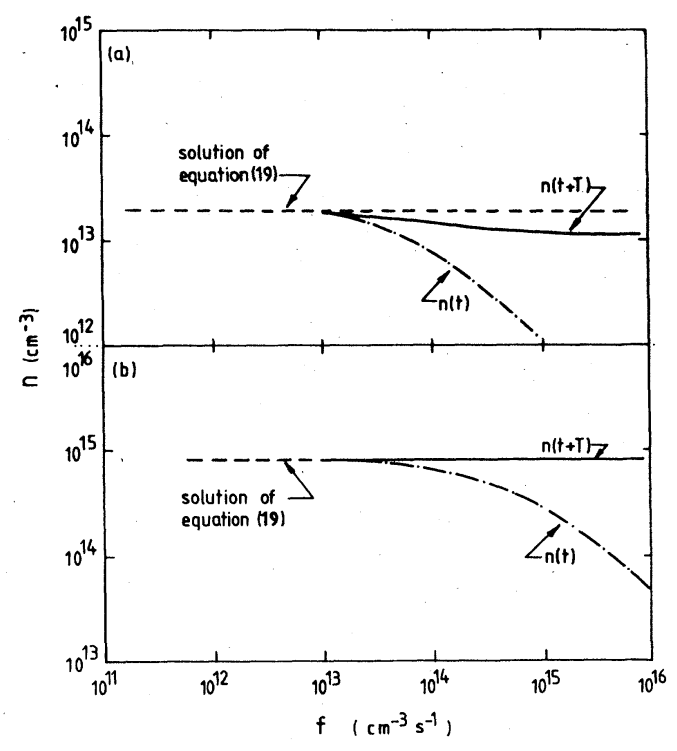

FIG. 11. Variation of $n(t+T)$ with $f$ for two sets of parameters. For (a) the parameters are $N=10^{15} \mathrm{~cm}^{-3}$; $N_{k}=10^{15} \mathrm{~cm}^{-3} ; N_{h}=10^{15} \mathrm{~cm}^{-3} ; A=10^{-16} \mathrm{~cm}^{3} \mathrm{~s}^{-1}$; $A_{k}=10^{-17} \mathrm{~cm}^{3} \mathrm{~s}^{-1} ; A_{r}=10^{-15} \mathrm{~cm}^{3} \mathrm{~s}^{-1} ;$ and $A_{h}=10^{-13}$ $\mathrm{cm}^{3} \mathrm{~s}^{-1}$. For (b) the parameters are the same except for $A=10^{-15} \mathrm{~cm}^{3} \mathrm{~s}^{-1}$ and $A_{r}=10^{-16} \mathrm{~cm}^{3} \mathrm{~s}^{-1}$. For both sets of curves the solution of Eq. (19) is the same as $n(t+T)$ and $n(t)$ at low $f$. For larger values of $f$, however, $n(t)$ deviates from this value and $n(t+T)$ only remains equal to the approximate solution in (b).

arrived at a set of inequalities which they contend must be obeyed in order to obtain a superlinear growth curve in the competing trap model. The inequalities are

$$
A_{k}>A_{r}
$$

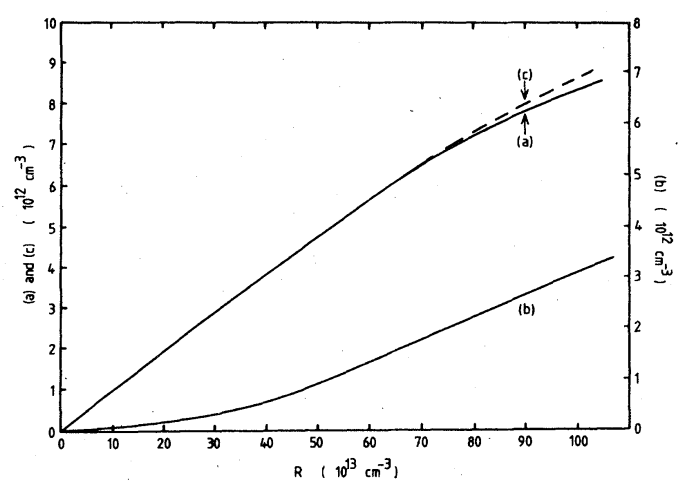

FIG. 12. A set of growth curves in which the solution of Eq. (19) agrees very closely with the values of $n(t+T)$. The parameters are the same as those for Fig. 11(b). (a) $n(t+T)$; (b) $n(t)$; (c) solution of Eq. (19). 


$$
\frac{N_{k} A_{k}^{2}}{N A}>A_{r},
$$

$$
A_{k}>A \text {. }
$$

We are now in a position to test these conditions more rigorously using the numerical analysis of Eqs. (14) - (18).

Typical growth curves obtained by choosing a set of parameters for which the inequalities given in Eqs. $(20 a)-(20 c)$ are obeyed are shown in Fig. 13. It can be seen that both $n(t)$ and $n(t+T)$ grow superlinearly. Three examples of growth curves, in which one or more of the inequalities given in Eqs. (20a) - (20c) are not obeyed are given in Figs. 14 - 16. In all cases superlinear growth is not seen and the shape of the $n(t+T)$ curve broadly parallels the solution of Eq. (19). In this respect the conditions for superlinearity, as determined by Chen and Bowman, appear to be correct. However, the agreement between the numerical and approximative solutions is only qualitative, not quantitative, as can be seen from a comparison of the curves shown in Figs. $14-16$. This lack of quantitative agreement is the result of the unsuitability of the condition of quasiequilibrium in these cases.

\section{DISCUSSION}

The purpose of this work has been to study theoretically the processes of trap filling such as the excitation of TL by ionizing radiation. Inasmuch as this work attempts to arrive at numerically exact solutions of otherwise intractable differential equations, it parallels the work of Kemmey et al..$^{14}$ and Kelly et al. ${ }^{15}$ and others who have numerically solved the corresponding differential

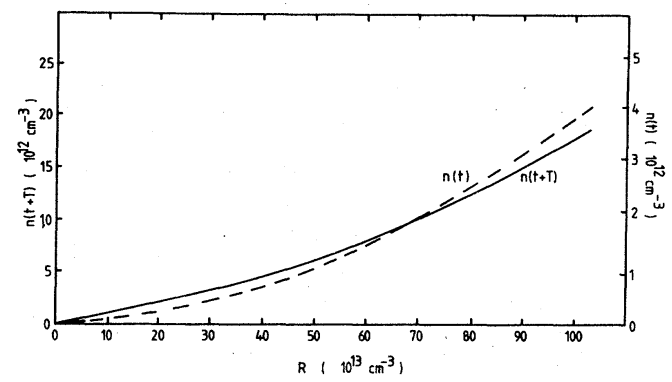

FIG. 13. Growth of $n(t+T)$ and $n(t)$ with $R$ for the two-traps - one-center case. The parameters' values are such that the inequalities given by Eqs. (20a) (20c) are obeyed, and both curves are seen to grow superlinearly. The parameters are the same as for Fig. 11 (b) but with $A=10^{-17} \mathrm{~cm}^{3} \mathrm{~s}^{-1}, A_{k}=10^{-15} \mathrm{~cm}^{3} \mathrm{~s}^{-1}$, and $A_{r}=10^{-13} \mathrm{~cm}^{3} \mathrm{~s}^{-1}$.

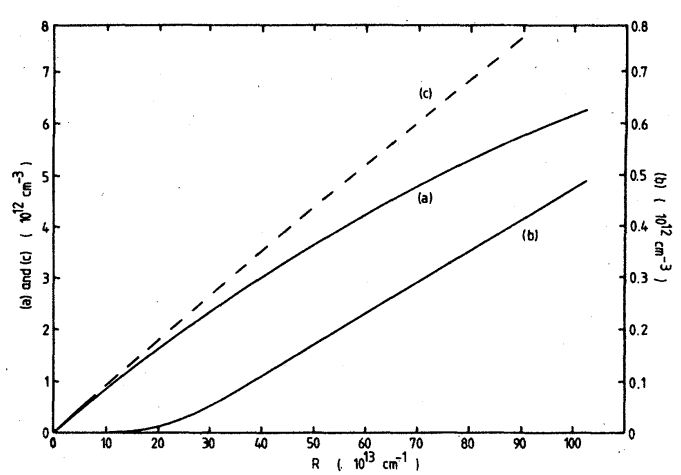

FIG. 14. An example of growth curves of (a) $n(t+T)$ and (b) $n(t)$ with $R$ for which one or more of the inequalities in Eqs. (20a) - (20c) are not obeyed. $n(t+T)$ does not exhibit superlinearity, but also does not agree quantitatively with either (c) or (b). The parameters chosen are the same as for Fig. 13, but with $A_{k}=10^{-16} \mathrm{~cm}^{3} \mathrm{~s}^{-1}$ and $A_{r}=10^{-16} \mathrm{~cm}^{3} \mathrm{~s}^{-1}$. (c) solution of Eq. (19).

equations for TL emission. The present work deals with trap filling (during irradiation) whereas these authors deal with trap emptying (during heating). Comparison of the numerical solutions with those resulting from analytical expressions produced by the introduction of approximations ${ }^{1-9}$ has shown that the exact applicability of the approximate solutions is very restricted.

A relatively simple model, having one or two electron traps and one or two hole recombination centers, has been considered. The model is by no means the only possibility for accounting for TL excitation since it disregards defect creation by the irradiation as well as thermal and athermal drainage during irradiation and since it assumes only a limited number of trapping states whereas more might be involved. There is no reason, however, to believe that making the model more complicated in order to make it more realistic, e.g., by

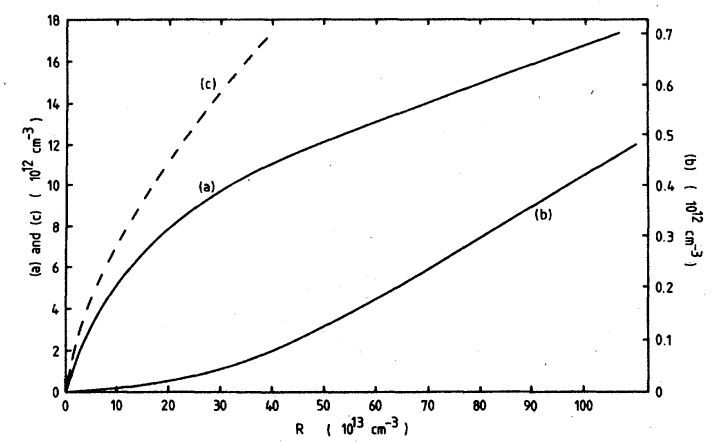

FIG. 15. As for Fig. 14, but with $A_{k}=10^{-16} \mathrm{~cm}^{3} \mathrm{~s}^{-1}$ and $A_{r}=10^{-16} \mathrm{~cm}^{3} \mathrm{~s}^{-1}$. 


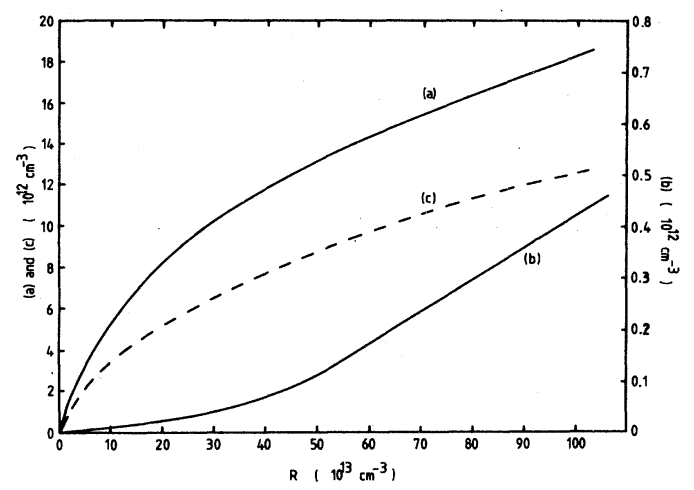

FIG. 16. As for Fig. 14, but with $A=10^{-16} \mathrm{~cm}^{3} \mathrm{~s}^{-1}$, $A_{k}=10^{-17} \mathrm{~cm}^{3} \mathrm{~s}^{-1}$, and $A_{r}=10^{-15} \mathrm{~cm}^{3} \mathrm{~s}^{-1}$.

allowing, say, for more traps and centers, will negate the main conclusions arrived at.

The addition of time $T$ (50 $\mathrm{s}$ as used by us) for simulating relaxation following excitation, is very important. Both in the one-trap - one-center and one-trap - two-center cases, it quite often happened that, say $n(t)$ went down with higher generation rates whereas $n(t+T)$ went up. It is certainly the latter which is relevant to the experimentally measured TL intensity.

This relatively simple model has been able to account for some recent experimental results. Groom et al. ${ }^{11}$ have reported upon a decrease in TL emission (of up to a factor of $\sim 5$ ) in the TL from natural quartz as the dose rate is increased over a certain range. The computational results indicate that such behavior is possible and indeed have produced variations of up to a factor of $\sim 20$. Furthermore, the additional observations by Valladas and Ferreira ${ }^{12}$ that the TL intensities of some spectral components of the TL from quartz decrease with increasing dose rate while others increase, can be simulated by the simple inclusion of an extra recombination center into the energy-level diagram.

A survey of the literature concerning TL and dose rate effects in various phosphors, reveals that a variety of results have been obtained regarding the dependence of the TL response upon dose rate. The results are summarized in Table $I^{11,12,16-21}$ In this table only a broad outline of the results obtained is described; for further details reference ought to be made to the relevant published articles.

It can be seen from the table that a wide range of values for both total dose and dose rate has been utilized experimentally in attempts to discover dose rate effects. Also, specimens from several different sources have been examined. In the light of the present theoretical analysis it is not surprising that there is such diversity in the results, sometimes even with the same host material (e.g., quartz). We have seen that the main factor leading to the dose rate dependence is that recombination occurs during excitation. This is very plausible since the recombination may very well be that which, during the heating of the sample, produces the light emission. Both TL decrease with increasing intensity and TL increase with increasing intensity - depending on the other parameters have been found. The nature of the effect (increase or decrease) was found to be particularly dependent on one of the parameters, $N_{h}$, in the one-trap one-center case. This may be the cause for the variety or even apparent irreproducibility of experimental results so far, since small changes in the effective concentration of a particular impurity or defect in the crystal may result from some procedural effects such as heating, exposure to a certain gas, or from the fact that the material (e.g., quartz) may originate from different sources [i.e., geological (e.g., Brazilian) or pottery].

At the same time, observations of dose rate-independent TL in certain dose rate ranges in various phosphors ${ }^{20,21}$ can also be encompassed by the computational results. Finding theoretically the dose rate dependence certainly does not mean that dose rate dependence should always be the case. The cases of the curves shown (e.g., Figs. 3 and 5) are only examples. In fact, all the calculated curves were dose rate independent at the high and low dose rate ranges. In this work we deliberately looked for ranges of parameters for which the dose rate effects are clearly seen. For other ranges of the relevant parameters, no effect or only a small effect was seen. Thus, the experimental resuIts summarized in Table I can all be embraced by the computational analysis.

It is worthwhile noting at this point that some previously unexplained observations ${ }^{22,23}$ may be due to similar dose rate effects to those described in this paper, but full attention has not been given to this aspect of the results in this earlier work. Additionally, in a recent paper Nilsson and Papsberg $^{24}$ observed a dose rate dependence for the yield of trapped electrons in crystalline ice. Although the treatment of the problem offered by these authors is broadly similar to that given in this paper, the physical processes considered by Nilsson and Papsberg differ from those considered here. Also, Nagpal ${ }^{25}$ reports the exposure rate dependence of TL from several types of phosphor irradiated with ultraviolet radiation, although once 


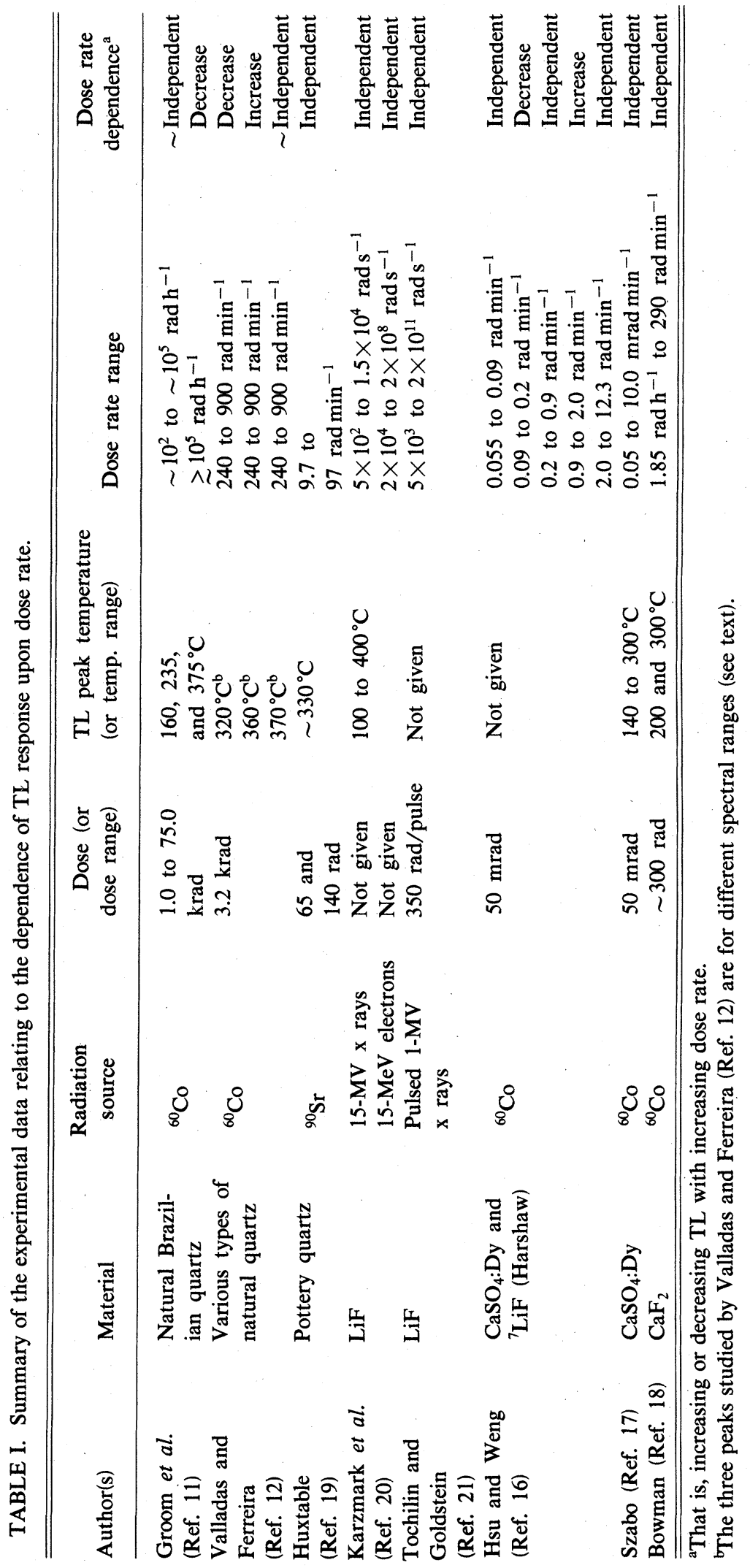


again the physical process may be different to that dealt with in this paper.

The amount of published work concerning the observation of superlinearity in TL-versus-dose curves is very large and it is unfeasible to collect the data together in a manner similar to that shown in Table I. Suffice it to say that the phenomenon of superlinear growth of TL with imparted dose is very common and that most of the commonly studied TL phosphors exhibit it, including LiF and quartz. Many mechanisms have been forwarded to explain superlinearity. The present analysis gives a detailed treatment of just one of the suggested mechanisms, namely, competition during irradiation. ${ }^{13}$

Comparing our dose dependence results to those of previous works, ${ }^{1-9}$ the main points are that we do not assume $n_{c}$ and $n_{v}$ to be small or to vary with time in any particular way. As compared to Maxia and colleagues, ${ }^{2-7}$ Aitken et al. ${ }^{1}$ and Bowman and Chen, ${ }^{8,9}$ we do not make the implicit assumption that the probability for trapping a hole from the valence band into the center is very high (which is expressed by $n_{v}$ being very small). One can thus get, at least in principle, the dose dependence for any set of given trapping parameters (al- though the program did not equally easily solve the equations for every set of chosen parameters).

It may look as a disadvantage that the final results cannot be shown as an analytical function, but rather as numerical results that can only be presented graphically. However, it is evident that the present results are much closer to the actual situation than the previous approximate results which can only serve as preliminary indications to the way the trap-filling process might behave.

We have shown that while studying the dose rate dependence, the total dose should be taken as a parameter and, in a similar way, while studying the dose dependence, the dose rate should be considered as a parameter. Although it may well be that for broad ranges of dose rates the dose dependence is independent of the dose rate, one should be alert to the possibility that this might occur while evaluating experimental results.

\section{ACKNOWLEDGMENT}

Financial support for this work was provided by the Science Research Council (UK).
*Permanent address: Department of Physics and Astronomy, Tel-Aviv University, Ramat-Aviv, Tel-Aviv, Israel.

${ }^{\dagger}$ Present address: School of Engineering and Applied Sciences, University of Sussex, Falmer, Brighton, Sussex, BN1 9QT, England.

${ }^{1}$ M. J. Aitken, J. Thompson, and S. J. Fleming, Proc. 2nd Int. Conf. Lumin. Dosimetry, Gatlinburg, Tenn. U.S. Atomic Energy Comm. CONF-680920 (1968).

${ }^{2}$ F. Aramu, V. Maxia, G. Spano, and C. Cortese, J. Lumin. 11, 197 (1975/76).

${ }^{3}$ F. Aramu, V. Maxia, and G. Spano, Lett. Nuovo Cimento 25, 75 (1975).

${ }^{4}$ V. Maxia, Lett. Nuovo Cimento 20, 443 (1977).

5V. Maxia, Phys. Rev. B 17, 3262 (1978).

${ }^{6}$ V. Maxia, Lett. Nuovo Cimento 24, 89 (1979).

7V. Maxia, Phys. Rev. B 21, 749 (1980).

${ }^{8}$ R. Chen and S. G. E. Bowman, European PACT J. $\underline{2}$, 216 (1978).

${ }^{9}$ S. G. E. Bowman and R. Chen, J. Lumin. 18/19, 345 (1979).

${ }^{10} \mathrm{R}$. Chen and Y. Kirsh, The Analysis of Thermally Stimulated Processes (Pergamon, Oxford 1981).

${ }^{11}$ P. J. Groom, S. A. Durrani, K. A. R. Khazal, and S. W. S. McKeever, European PACT J. 2, 200 (1978).
${ }^{12}$ G. Valladas and J. Ferreira, Proc. 6th Int. Conf. Sol. St. Dosim., Toulouse: [Nucl. Instrum. Methods 175, 216 (1980)].

${ }^{13}$ N. Suntharalingam and J. R. Cameron, Phys. Med. Biol. 14, 397 (1969).

14P. J. Kemmey, P. D. Townsend, and P. W. Levy, Phys. Rev. 155, 917 (1967).

15P. Kelly, M. J. Laubitz, and P. Bräunlich, Phys. Rev. B 4, 1960 (1971).

16P. C. Hsu and P. S. Weng, Nucl. Instrum. Methods 138, 307 (1976).

17P. P. Szabó, Nucl. Instrum. Methods 147, 451 (1977).

${ }^{18}$ S. G. E. Bowman, Proc. Specialist Seminar on TL Dating, Oxford, 1980. [European PACT J. (in press)].

19J. Huxtable, Ancient TL 9, 9 (1979).

${ }^{20}$ C. J. Karzmark, J. White, and J. F. Fowler, Phys. Med. Biol. 9, 273 (1964).

${ }^{21}$ E. Tochilin and N. Goldstein, Health Phys. 12, 1705 (1966).

${ }^{22}$ A. Halperin and R. Chen, Phys. Rev. 148, 839 (1966).

${ }^{23}$ M. C. Wintersgill and P. D. Townsend, Radiat. Eff. 38, 113 (1978).

${ }^{24}$ G. Nilsson and P. Papsberg, Chem. Phys. Lett. 4, 119 (1980).

25J. S. Nagpal, Rad. Effects Lett. 4ㄱ, 173 (1979). 